
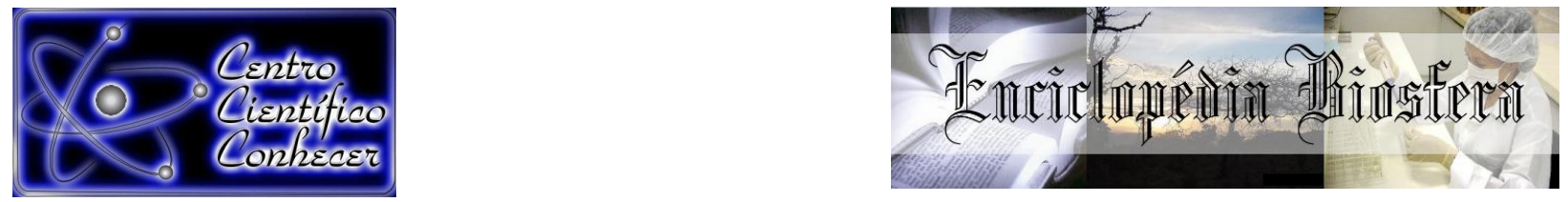

\title{
REAÇAO DE GENÓTIPOS DE SOJA AO NEMATÓIDE DAS LESOES RADICULARES
}

Rayane Gabriel da Silva ${ }^{1}$ Tânia de Fátima Silveira dos Santos ${ }^{2}$, Igor Cezar Duarte da Silva ${ }^{3}$, Mickael Bruno Saraiva da Silva ${ }^{4}$ Guilherme Augusto Neris Borges ${ }^{5}$

\author{
135 Graduando (a) em Agronomia, Faculdade Anhanguera Rondonópolis (FAR) \\ Rondonópolis, Brasil E-mail: rayane.agro@gmail.com \\ ${ }^{2}$ Gerente técnica de Laboratório APROSMAT \\ ${ }^{4}$ Auxiliar de casa de vegetação - APROSMAT
}

Recebido em: 08/09/2015 - Aprovado em: 14/11/2015 - Publicado em: 01/12/2015
DOI: http://dx.doi.org/10.18677/Enciclopedia_Biosfera_2015_219

\section{RESUMO}

Nos últimos anos, os nematóides das lesões radiculares (Pratylenchus brachyurus), têm causado significativas perdas na cultura da soja, sobretudo, em regiões do Centro-oeste. Entretanto, são poucas as informações de genótipos de soja resistentes à infestação do nematoide das lesões radiculares. Objetivo do trabalho foi avaliar a reação de dezoito genótipos de soja infectados com população de Pratylenchus brachyurus. O delineamento experimental utilizado foi inteiramente casualizado (DIC), constituído por dezenove tratamentos e 6 repetições. Os genótipos estudados foram: W791RR, M9144, GB 881RR, P98Y11 RR, ST820 RR, MGBR CONQUISTA, BRSMT Pintado, TMG 4182, M 7639, Tabarana, ST810 RR, NA8015 RR, TMG1179 RR, TMG 4190, TMG115 RR, TMG1176 RR, W851 RR, TMG1288 RR. A Crotalária spectabilis foi incluída como padrão de resistência a $P$. brachyurus. Cada parcela foi constituída por vasos de argila, com capacidade de 1 $\mathrm{dm}^{3}$ de solo contendo mistura de solo: areia (1:3), esterilizadas. Constatou-se que todos os materiais foram suscetíveis ao nematoide, apresentando fator de reprodução de 2,30 a 18,42. Os genótipos W791 RR com FR $(2,30)$ e M $9144(2,42)$ foram os menores FR obtidos, entretanto, permitiu a multiplicação dos nematoides.

PALAVRAS-CHAVE: Genótipos, Glycines max, Pratylenchus brachyurus.

\section{SOYBEAN GENOTYPES REACTION TO LESION NEMATODE ROOT.}

\begin{abstract}
In recent years, the nematodes of root lesions (Pratylenchus brachyurus), have caused significant losses in soybean, especially in West-Central regions. However, there is little information soybean genotypes resistant to the nematode infestation of the root injury. Work objective was to evaluate the reaction of eighteen soybean genotypes infected with a population of Pratylenchus brachyurus. The experimental design was completely randomized (DIC), consisting of nineteen treatments and 6 repetitions. The genotypes studied were: W791RR, M9144, GB 881RR, P98Y11 RR, ST820 RR, MGBR CONQUEST, BRSMT Pintado, TMG 4182, M 7639, Tabarana, ST810 RR, NA8015 RR, TMG1179 RR, TMG 4190, TMG115 RR, TMG1176 RR, W851 RR, RR TMG1288. The Crotalaria spectabilis was included as standard to the nematode resistance. Each plot consisted of clay vessels, with a capacity of $1 \mathrm{dm} 3$ of soil containing soil mix: sand (1: 3 ), sterilized. It was found that all materials are susceptible to the nematode, with a reproduction factor of the 18.42 2.30. The W791
\end{abstract}


RR genotypes with FR (2.30) and M 9144 (2.42) were the smallest FR obtained, however, allowed the proliferation of nematodes.

KEYWORDS: Genotypes, Glycines max, Pratylenchus brachyurus.

\section{INTRODUÇÃO}

A cultura da soja (Glycine max L. Merril), tem se expandido em grande escala comercial em todo país, em especial nas regiões Centro-Oeste, devido às boas condições de clima e adaptabilidade das cultivares disponíveis. A área cultivada em conjunto com alto nível tecnológico, resulta em 11,46 milhões de hectares plantados (CONAB, 2012), caracterizando a importância comercial da cultura.

Com a expansão de novas áreas cultivadas no Estado de Mato Grosso, os problemas fitossanitários se intensificaram, especialmente os relacionados aos fitonematoides parasitas de plantas. Os nematoides foram favorecidos por mudanças no sistema de produção e instabilidade das culturas. Além da soja, podem parasitar varias espécie de plantas cultivadas, coberturas verdes e a maioria das plantas daninhas (RIBEIRO et al., 2009), sendo que a ultima, servem como hospedeiras na entressafra para a conservação desse patógeno no sistema.

O nematoide das lesões radiculares (Pratylenchus. brachyurus) posiciona-se como o mais importante nematoide da agricultura (LORDELLO, 1985). Entretanto, as informações sobre os efeitos do seu parasitismo nas diferentes culturas são escassa e as perdas ocasionadas para a cultura da soja, caracterizada como a mais plantada, são variados. O parasitismo do $P$. brachyurus está distribuído por toda parte no estado, estando presente em boa parte das plantações de soja, com ocorrência em 96\% das amostras coletadas (RIBEIRO, 2010). De acordo com o grau de infestação há relatos frequentes de reduções na produção de $30 \%$ podendo alcançar até 50\% (GOULART, 2008; DIAS et al., 2010) em lavouras comerciais.

O sistema de produção vigente no Estado complica os programas de manejo, pois, dependendo do grau de suscetibilidade das coberturas vegetais aos nematoides, as populações desses fitoparasitas tendem a aumentar, atingindo densidades populacionais altas podendo prejudicar as culturas de verão (SILVA, 2011). Em razão das diversas características existentes do nematoide, seu controle se torna atividade complexa, sendo que, após a infestação da área, a sua erradicação é praticamente impossível (FERRAZ et al., 2010).

O controle de nematoide frequentemente utilizado é o método químico, cultural e genético. O controle químico feito com o uso de nematicidas é uma ferramenta eficaz, mas oferece certas limitações como: alta toxicidade, risco de contaminação ambiental, alto custo e baixa disponibilidade em países em desenvolvimento e/ou baixa eficácia de controle depois de repetidas aplicações (DONG \& ZHANG, 2006), e não substitui estratégias de manejo como rotação de culturas, pousio, plantas antagonistas e cultivares resistentes (FERREIRA, 2010).

Outra prática com potencial para o controle de $P$. brachyurus, mas vem sendo pouco estudada, é o pousio, que consiste em manter o solo por certo período sem qualquer vegetação, de preferência também com revolvimento por meio de aração elou gradagem. Dessa forma, os nematoides morrem por ausência de hospedeiro, ou por dessecação pela ação da luz (INOMOTO, 2008). Contudo, foge dos padrões conservacionistas de solos, e implica na condenação da implantação do sistema plantio direto (SPD).

De acordo com ROBERTS (2002), a resistência genética de plantas é um dos métodos mais eficientes e econômicos de evitar as perdas ocasionadas pelos nematoides. A resistência descreve a habilidade da planta em suprimir ou restringir a 
multiplicação dos nematóides (FERRAZ et al., 2010), mesmos em áreas com populações superiores ao nível de infestação.

O melhoramento genético visando incorporar resistência a $P$. brachyurus é complexo, e necessita de varias técnicas para manter a resistência da planta quando parasitada por este patógeno, que por ser polífago, pouco especializado, e de hábito endoparasita migrador (GOULART, 2008), podem abandonar as plantas parasitadas, em busca de novos hospedeiros. Entretanto se torna a opção mais favorável para reduzir o nível populacional do patógeno, inibindo a penetração e multiplicação do nematoide no hospedeiro.

Haja visto a necessidade de cultivares de soja resistente a Pratylenchus brachyurus e a carência desses materiais disponíveis no mercado, o presente trabalho teve como objetivo avaliar a reação de dezoito genótipos de soja infectadas com população de Pratylenchus brachyurus em ambiente controlado.

\section{MATERIAL E MÉTODOS}

O experimento foi conduzido em casa-de-vegetação da APROSMAT, em Rondonópolis, MT, (latitude $16^{\circ} 28^{\prime} 15^{\prime \prime} \mathrm{S}$ ) no período de Setembro a Dezembro de 2013.

O delineamento experimental utilizado foi inteiramente casualizado (DIC), constituído por dezenove tratamentos e seis repetições. As cultivares de soja avaliados quanto à reação dos nematoides das lesões $P$. brachyurus foram: W791RR, M9144, GB 881RR, P98Y11 RR, ST820 RR, MGBR CONQUISTA, BRSMT Pintado, TMG 4182, M 7639, Tabarana, ST810 RR, NA8015 RR, TMG1179 RR, TMG 4190, TMG115 RR, TMG1176 RR, W851 RR, TMG1288 RR. A Crotalária spectabilis foi incluída como padrão de resistência ao nematoide das lesões.

Os materiais foram semeados em vasos de argila com capacidade de $1 \mathrm{dm}^{3}$ de solo, contendo mistura de solo: areia (1:3) esterilizada. Sete dias após a semeadura, as plântulas foram desbastadas, permanecendo apenas uma planta por vaso, em seguida foram inoculadas na rizosfera da plântula. Os vasos foram mantidos em casa-de-vegetação em temperatura de $25-35^{\circ} \mathrm{C}$, com irrigação diária.

O inoculo inicial de Pratylenchus brachyurus foi adquirido a partir de populações puras, perpetuada em genótipo de quiabo santa cruz para extração dos espécimes (juvenis e adultos) do nematoide. Para isto foi adotada a metodologia de Boneti \& Ferraz (1981). A inoculação foi permitida através de dois orifícios de $2 \mathrm{~cm}$ de profundidade, próximos as radicelas das plântulas a pipetagem de volumes préajustados da suspensão contendo os nematóides. A população inicial ( $\mathrm{Pi})$ foi verificada através da técnica de (Jenkins1964), constatando-se Pi de aproximadamente 600 espécimes.

Aos 75 DAS, as partes aéreas foram descartados, os sistemas radiculares cuidadosamente retirados dos vasos, logo lavados para a retirada do solo adjacente, em seguida, fragmentados em pedaços com auxílio de tesoura. As raízes foram trituradas em liquidificador doméstico passando-as, em sequência, as suspensões resultantes da trituração através das peneiras de 60 e 400 mesh, para a extração dos juvenis e adultos de P.brachyurus, o material resultante da peneira de 400 mesh foram então removidas com auxilio de piceta e vertida em becker de $100 \mathrm{ml}$. Posteriormente, seguiram para identificação e quantificação dos nematoides em microscópio estereoscópico e câmara de Peters.

Os cálculos dos FR (Fatores de reprodução) foram atingidos por meio de a fórmula descrita a seguir: $\mathrm{FR}=\mathrm{Pf} / \mathrm{Pi}$ (população final/população inicial) estabelecido pela quantidade entre o número de ovos/J2 por sistema radicular somados 
(população final) e o número de ovos/J2 utilizados no inoculo (população inicial), segundo Oostenbrink (1966) FR superiores ou iguais a 1,0 representam plantas suscetíveis e FR inferiores a 1,0 indicam plantas resistentes.

Os dados obtidos nos ensaios foram submetidos à análise de variância pelo teste de $\mathrm{F}$ (tabela 1) a $5 \%$ de probabilidade e, quando houve diferença significativa entre os tratamentos, suas médias foram comparadas pelo teste de Tukey (FERREIRA, 2007).

\section{RESULTADOS E DISCUSSÕES}

Os resultados referentes à reprodução do de $P$. brachyurus, foram expressos em FR, mostrando grande variação entre as cultivares de soja utilizadas no ensaio, cujos valores variaram de 2,30 a 18,42 (Tabela 2). As linhagens W 791 RR e M-9144 obtiveram menor FR, sendo 2,30 e 2,42 Contudo, são cultivares que permitem a sobrevivência e multiplicação do nematoide em menor escala, entretanto, são opções para serem plantadas em aéreas de baixas e médias populações, haja visto que são poucas as cultivares de soja resistente a $P$. brachyurus.

De acordo com os dados, todas as cultivares de sojas estudadas permitiram a multiplicação de P.brachyurus, sendo materiais que apresentaram FR superiores a $(1,00)$ que segundo Oostenbrinks,(1996) para a cultivar ser considerada resistente devem apresentar FR menores que 1,00, já as cultivares suscetíveis são os que forem superiores a 1,00 , iguais os resultados obtido nesse trabalho.

TABELA 1. Valor de $F$ obtido na análise de variância ${ }^{11}$ para fator de reprodução (FR) de Pratylenchus brachyurus em função dos genótipos de soja.

\begin{tabular}{lcc}
\hline Fonte de variação & GL & FR \\
\hline Tratamento & 18 & $3,84^{* *}$ \\
Resíduo & 76 & - \\
Total & 94 & - \\
\hline CV $(\%)$ & - & 34,64 \\
\hline
\end{tabular}

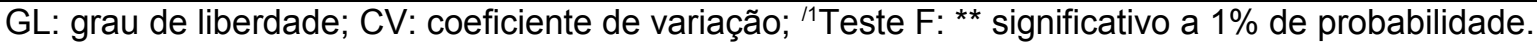

TABELA 2: Distribuição dos genótipos e respectivos fatores de reprodução (FR).

\begin{tabular}{ccc}
\hline Tratamentos & Genótipos & $\begin{array}{c}\text { Fator de Reprodução } \\
\text { (FR) }\end{array}$ \\
\hline 1 & Crotalaria spectabilis & $0,06 \mathrm{a}$ \\
2 & W 791 RR & $2,30 \mathrm{ab}$ \\
3 & M-9144 & $2,42 \mathrm{ab}$ \\
4 & GB 881 RR & $3,90 \mathrm{abc}$ \\
5 & P 98Y11RR & $4,94 \mathrm{abc}$ \\
6 & ST 820 RR & $5,12 \mathrm{abc}$ \\
7 & MG BR CONQUISTA & $5,76 \mathrm{abc}$ \\
8 & BRS MT PINTADO & $5,2 \mathrm{abc}$ \\
9 & TMG 4182 & $5,84 \mathrm{abc}$ \\
10 & M 7639 & $7,38 \mathrm{abc}$ \\
11 & TABARANA & $8,26 \mathrm{abc}$ \\
12 & ST 810 RR & $10,60 \mathrm{abc}$ \\
13 & NA 8015 RR & $8,68 \mathrm{abc}$ \\
14 & TMG 1179 RR & $9,28 \mathrm{abc}$ \\
15 & TMG 4190 & $11,34 \mathrm{bc}$ \\
17 & TMG 115 RR & $11,00 \mathrm{bc}$ \\
18 & TMG 1176 RR & $16 \mathrm{bc}$ \\
19 & W 851 RR & $18,42 \mathrm{c}$ \\
probabilidade. & TMG 1288 RR & $18,42 \mathrm{c}$ \\
\hline Médias & mas colunas não diferem pelo teste de Tukey a $5 \%$ de &
\end{tabular}


Segundo RIBEIRO et al., (2007), (FR) relativamente baixos para $P$. brachyurus na faixa de 1,2 a 4,0 são valores significativamente menores que os verificados nas cultivares suscetível (FR 20 a 30). Embora baixos, situam-se acima de 1 e portanto permitem o aumento populacional do nematoide mesmo em menores escalas sendo suscetivel.

A cultivar MGBR Conquista obteve o FR de 5,76 (tabela 02), divergindo de Santos, (2012) que testou esta mesma cultivar e obteve FR de 0,47 classificando-a como resistente, contudo SILVA (2011) também testou esta cultivar em diferentes níveis de inoculo e obteve variabilidade nos resultados $50(1,30), 75(2,67), 250$ $(1,54)$ e $500(0,12)$, corroborando com os dados expressos neste estudo.

SANTOS , (2012) também testou a cultivar TMG 115 RR e obteve FR $(1,28)$, considerando a mesma, uma cultivar suscetível aos danos do nematoide, resultado este que evidencia a representatividade dos resultados avaliados neste experimento, FR $(11,00)$.

Os resultados obtidos corroboram os dados obtidos por COSTA \& FERRAZ (1998), nas quais testaram diferentes cultivares e linhagens de soja UFV e IAC e todos foi suscetível à multiplicação de $P$. brachyurus, apresentando \% de FR 2,6 a 56,14 . Entretanto, esses estudos revelam a carência de se obter genes com resistência a esse nematoide.

\section{CONCLUSÕES}

Conclui-se que todas as cultivares de soja testadas nesse trabalho foram suscetíveis ao nematoide das lesões radiculares, porém os genótipos W791 RR com FR $(2,30)$ e M 9144 (2,42 ) foram os menores FR encontrados.

A Crotalária spectabilis pode ser adotada no manejo de $P$. brachyurus, pois seu fator de reprodução do nematoide no sistema radicular foi inferior a 1,0 , contatando a resistência a $P$. brachyurus.

\section{REFERÊNCIAS}

COSTA, D. C. \& FERRAZ, S. Avaliação da resistência de cultivares e linhagens de soja a Pratylenchus brachyurus. Pesquisa Agropecuária Tropical, Goiânia, v. 28, n. 2, p. 67-76, 1998.

CONAB - Companhia Nacional de Abastecimento. Acompanhamento da safra 2011/2012: Décimo levantamento. Brasília: Ministério da Agricultura, 2012. 28p.

BONETI, J. I. S. \& S. FERRAZ. Modificação do método de Hussey e Barker para extração de ovos de Meloidogyne exígua de raízes de cafeeiro. Fitopatologia Brasileira, 6: 553.1981.

DIAS, W. P.; ORSINI, I. P.; RIBEIRO, N. R.; PARPINELLI, N. M. B.; FREIRE, L.L.; Hospedabilidade de plantas daninhas a Pratylenchus brachyurus. Anais, XXX Congresso Brasileiro de Nematologia, Uberlândia, MG, 2012.

DIAS, W.P.; ASMUS, G. L.; SILVA, J.F.V.; GARCIA, A.; CARNEIRO, G.E.S. Nematoides. In: Almeida, A. M. R.; Seixas, C. D. S. (Ed.) Soja: doenças radiculares e de hastes e inter-relações como manejo do solo e da cultura. Embrapa Soja: Londrina, p. 173-206. 2010. 
DONG, L. Q.; ZHANG, K. Q. Microbial control of plant-parasitic nematodes: a fiveparty interaction. Plant Soil, v. 288, n. 1, p. 31-45, 2006.

FERREIRA, D. F. Sisvar - Sistema de análise de variância. Versão 5.3. Lavras: UFLA, 2007.

FERRAZ, S.; FREITAS, L. G.; LOPES, E. A.; DIAS-ARIEIRA, C.R. Manejo Sustentável de Fitonematoides. Viçosa, MG, Ed. UFV, 2010. 306 p.

GOULART, A. M. C. Nematoides das lesões radiculares (Gênero Pratylenchus). 2008. Disponível em: http://www.agrosoft.org.br/agropag/103613.htm. Acesso em 30 julho.2015

INOMOTO, M. M. Importância e manejo de Pratylenchus brachyurus. Revista Plantio Direto, v. 108, p. 4-9, 2008.

JENKINS, W.R. A rapid centrifugal-flotation technique for separating nematodes from soil. Plant Disease Reporter 48:692. 1964.

LORDELLO, L. G. E. Nematóides das plantas cultivadas. São Paulo: Nobel, 1985.

OOSTENBRINK, M. Major characteristics of the relation between nematodes and plants. Mededelingen Landbouw, v.66, n.4, p.1-46, 1966.

RIBEIRO, N. R. Dias, W. P., Homechin, M., Silva, J. F. V. \& Francisco, A. Reação de genótipos de soja a Pratylenchus brachyurus. Nematologia Brasileira 31: 157158.2007b.

RIBEIRO, R. N.; et al. Reação de genótipos de soja a $P$. brachyurus. In: CONGRESSO BRASILEIRO DE NEMATOLOGIA, 27, 2007, Goiânia, Resumos... Piracicaba: Nematologia Brasileira, 2007b. p. 157-158.

RIBEIRO, N. R. Avaliação de espécies vegetais e cultivares de soja para a composição de esquemas de rotação ou sucessão de culturas para o manejo de Pratylenchus brachyurus. 2009. $56 \mathrm{f}$ Tese (Doutorado em Agronomia) Universidade Estadual de Londrina, Centro de Ciências Agrárias, Londrina, 2009.

RIBEIRO, N. R.; DIAS, W. P.; SANTOS, J. M. Distribuição de fitonematoides em regiões produtoras de soja do estado de Mato Grosso. Boletim de Pesquisa de Soja , Fundação Mato Grosso - p. 289-296.2010.

ROBERTS, P. A. Concepts and consequences of resistance. In: STARR, J. L.; COOK, R.; BRIDGE, J. (Eds.). Plant resistance to parasitic nematodes. Wallinghord: CAB International, p. 23-41.2002.

SANTOS, T. F. S. Reação de genótipos de soja aos nematoides das galhas e das lesões. 2001. 85 f Dissertação (Mestrado em Engenharia Agrícola), Universidade Federal de Mato Grosso, Rondonópolis. 2012. 
SILVA, R.B. Interação entre diferentes cultivares níveis populacionais iniciais de Pratylenchus brachyurus e cultivares de soja no desenvolvimento da planta e na reprodução do nematoide. 2011. 39 f. Dissertação (Mestrado em Agronomia/Fitopatologia) -Universidade Federal de Uberlândia- Uberlândia. 2011. 